ISSN: 0210-7287

DOI: http://dx.doi.org/10.14201/161620199287315

\title{
¿QUÉ ES UN MUNDO? ¿QUÉ ES UNA LITERATURA MUNDIAL? UNA DISCUSIÓN ${ }^{1}$
}

\section{What Is a World (Literature)? A Conversation}

\author{
Pheng CHEAH \\ University of California, Berkeley \\ pcheab@berkeley.edu
}

David DAMROSCH

Harvard University

ddamrosch@fas.harvard.edu

\author{
Traductor: César DOMÍNGUEZ \\ Universidade de Santiago de Compostela \\ cesar.dominguez@usc.gal
}

Se ofrece aquí la transcripción en traducción al castellano de la presentación por Pheng Cheah de su libro What Is a World? On Postcolonial Literature as World Literature (Cheah 2016) seguida de su discusión con David Damrosch, así como una selección del turno de preguntas y respuestas. Este evento tuvo lugar en la sesión de 2018 del Instituto de Literatura Mundial, con sede en la Universidad de Harvard, que acogieron el Catedrático Mitsuyoshi Numano y el Departamento de Estudios Literarios Contemporáneos de la Universidad de Tokio.

1. Pheng CheAH y David DamrosCH, "What Is a World (Literature)?», Journal of World Literature 4, n. ${ }^{\circ} 3$ (Agosto 2019): 305-329. Traducción de César Domínguez. Texto traducido con la autorización de los autores y los editores de la revista. 
Pheng CheAH: Permítanme que comience indicando que el título de mi libro What Is a World?. On Postcolonial Literature as World Literature alude al libro de David Damrosch (2003) What Is World Literature? de forma muy clara. Aprendí mucho con el libro de David, que, si no ha inaugurado un nuevo campo en los estudios literarios, ciertamente les ha dado un nuevo ímpetu y una nueva vida. No habría escrito mi libro si no hubiese leído el suyo. La pregunta que quería plantear es como un prolegómeno a la pregunta formulada por el libro de David, a saber, cuando hablamos de literatura mundial, pero también de campos relacionados, como historia mundial, cine mundial, religión mundial, arte mundial o música mundial, ¿qué entendemos exactamente por el término mundo? Como le dije a David por correo electrónico cuando tan amablemente me invitó a esta discusión con él, mi interés por la worldliness (mundanalidad) viene de tiempo atrás y es previo a mi interés por la literatura mundial, ya que, a diferencia de casi todos quienes discuten sobre literatura mundial recientemente, yo no enseño en un departamento de literatura ${ }^{2}$.

Mi interés por la mundanalidad procede de un interés previo por el cosmopolitismo, sobre el que he escrito algunas cosas. Dado que mi tesis doctoral versó sobre crítica literaria, los colegas en el campo de los estudios literarios no dejan de preguntarme acerca de la relación entre cosmopolitismo y literatura mundial. Inicialmente, no me interesó este tema. Mi trabajo sobre el cosmopolitismo deriva de interrogantes que están más próximos de la filosofía política y moral, las ciencias sociales y el derecho internacional público. Pero no pude responder acerca del porqué de mi desinterés, por lo que carraspeaba y murmuraba molesto. Finalmente, decidí escribir este libro, en parte en respuesta a las investigaciones sobre literatura mundial de finales de la década de 1990 y principios de la de 2000, que procuraban vincularla con las dinámicas globales y llevarla más allá de la idea desfasada de las grandes obras que conforman la herencia literaria de la humanidad, a partir de los artículos de Franco Moretti (2000 y 2003) en New Left Review, el libro de Pascale Casanova (2001) La República mundial de las Letras, que apareció originalmente en francés cuando yo era un doctorando y acerca del cual mi maestro Benedict Anderson estaba muy entusiasmado y, por supuesto, el libro de David.

Aquello que me sorprendió acerca de estas nuevas teorías sobre la literatura mundial fue que el término clave no fuese objeto ni de especificaciones

2. Traduzco worldliness por mundanalidad en el sentido de la primera parte de su definición por el DEL (actualización 2019), esto es, 'perteneciente o relativo al mundo como sociedad humana'. [Nota del trad.] 
concretas ni de examen, y que tampoco se definiera rigurosamente. Definían la literatura mundial como algo opuesto a la literatura nacional y la vinculaban a la globalización. De manera que, en primera instancia, era un término descriptivo, en el que mundial se identifica con global. Era "literatura global" lo que tenían en mente, literatura que circulaba globalmente. Pero dado que «literatura global» no parecía un término refinado, se recurrió a "literatura mundial", con su referencia al proyecto normativo de Goethe acerca de la Weltliteratur.

Fue entonces cuando pensé un poco más sobre esto y en un momento de mea culpa me di cuenta de que si uno revisa los trabajos sobre cosmopolitismo, incluido el mío, aunque hay una comprensión más elaborada del concepto mundo en comparación con «literatura mundial» y mundo es visto como un término normativo que significa un tipo de proyecto con un horizonte ideal, pese a todo el discurso sobre el cosmopolitismo se vuelve dogmático, porque hay algo que se da por hecho, a saber, la ecuación entre mundo y relaciones intersubjetivas humanas. Porque si el mundo se constituye a través de la circulación, son los sujetos humanos quienes ponen las cosas en circulación. A este respecto, los puntos de referencia son la filosofía griega, el pensamiento ilustrado francés, Kant y Marx. Si dejamos momentáneamente de lado la complejidad del concepto griego kosmos, que no es idéntico al latino mundus, y nos centramos fundamentalmente en la idea moderna de mundo, entonces mundo es el trato humano universal, sea que se piense en él como trato literario o como el trato material del comercio. En este último sentido, Marx afirmó que la historia mundial y la literatura mundial solo emergen cuando hay un mercado mundial. Pero, entonces, ¿por qué debemos dar por sentado que el mundo está constituido por el trato humano?

Así que mi interés por la mundanalidad no era, en primera instancia, de tipo ético-político en cuanto a sus motivaciones, ni estaba relacionado con la postcolonialidad. La mundanalidad es, para decirlo con un término pasado de moda, una cuestión ontológica. Es un objeto de la mayor extensión espacial posible que sirve como contenedor de todos los objetos y sujetos. Aquello que es mundial en la literatura es su locomoción en un espacio de Mercator de acuerdo con las coordenadas matemáticas de la geometría euclidiana. De forma paralela, la literatura mundial es el intercambio o circulación de un objeto entre sujetos, su movimiento a través de una distancia espacial plana en el tiempo concebido espacialmente. Pero, en su definición convencional como la ampliación de nuestro círculo de pertenencia desde la familia a la aldea, de la nación al mundo, el cosmopolitismo implica un movimiento hacia fuera que supera y trasciende las barreras territoriales. El trato mundial trasciende las fronteras del estado territorial y la comunidad imaginada de la nación. De ello se sigue que el 
cosmopolitismo de la literatura mundial consiste en el impacto de dichos movimientos espaciales sobre la producción, recepción e interpretación de los textos literarios. La mundanalidad de la literatura hace referencia a la escala global de producción, circulación, consumo y evaluación de la literatura, todo ello entendido sobre la analogía del alcance del mercado global.

En mi opinión, hay dos problemas con esta comprensión espacial de la mundanalidad. En primer lugar, hay que tomar en consideración que el mundo es originalmente una categoría temporal, de la que se deriva su dimensión normativa. Lo mundano hace referencia a la condición temporal de una existencia finita frente al carácter infinito o absoluto de la divinidad. La vocación normativa de la humanidad mundana es trascender la particularidad finita. Esta trascendencia es la esencia del proyecto espiritual de Goethe de una Weltliteratur que aspira a hacer emerger una humanidad universal mediante sus expresiones literarias específicas. En las manos de alguien como Erich Auerbach, la literatura mundial se conectaba necesariamente con una teleología de la historia mundial. Por el contrario, la reciente ecuación entre mundanalidad y circulación global de las obras literarias soslaya la dimensión temporal del mundo y achata el carácter horizontal de la literatura mundial. En consecuencia, solo permanece una normatividad mínima en los estudios recientes sobre la literatura mundial: la superación de la barreras territorial-nacionales. De hecho, en la comprensión fenomenológica de la mundanalidad, la espacialización del mundo epitomizada por la globalización es un proceso de desmundanización, Entweltlichung. Destruye la mundanalidad del mundo al reducirla a un globo ${ }^{3}$.

En segundo lugar, entender la literatura mundial en términos de circulación global implica que los procesos literarios reflejan los procesos globales. En el caso de Casanova o de Moretti, esto significa que la literatura tiene, en el mejor de los casos, un poder causal o formativo muy débil en relación con el mundo. Resuena aquí la caracterización por parte de Marx de las formaciones del espíritu como superestructuras de segundo grado, superestructuras de la superestructura legal y política del estado burgués. En consecuencia, se pierde el poder de formación de mundo que las teorías normativas atribuyen a la literatura mundial como medio de actualizar la humanidad y humanizar el mundo existente.

Por el contrario, si se contempla el mundo como una categoría temporal en vez de un objeto espacial, esto es, como un proceso de mundanización,

3. Esto puede explicar por qué no ha habido una reflexión sobre literatura mundial y cosmopolitismo, excepto por la sugerencia de que los textos literarios generan apegos múltiples a través de procesos de circulación. 
entonces puede afirmarse que la literatura posee una conexión fundamental con la mundanalidad. Por ejemplo, los filósofos de la tradición fenomenológica -Heidegger, Arendt, Derrida- le otorgaron a la literatura un papel privilegiado en sus explicaciones sobre el mundo. Para Heidegger, la poesía revela la mundanalidad del mundo. Para Arendt, el teatro es el mejor ejemplo de creación de mundo como intersticialidad subjetiva de palabras y hechos, lenguaje y acción. Y para Derrida, la narración está ligada a la apertura del mundo por el don del tiempo. En otras palabras, la literatura no cartografía meramente el mundo espacializado y le da valor y significado, sino que más bien sus estructuras formales promulgan la apertura de un mundo a través del darse y el venir del tiempo. Esto constituye un antídoto en contra de relegar la literatura a una infraestructura oscura. Podemos decir así que el mundo posee una estructura "literaria" que es más infraestructural, para hacer uso del lenguaje marxista, que la realidad material de la producción económica.

Comprender la literatura mundial en términos de la conexión de la literatura con la mundanalidad y el venir del tiempo apunta a recursos inmanentes para resistir la globalización capitalista. La acumulación capitalista necesita y requiere tiempo. El capital aumenta mediante tecnologías y cálculos racionales que se apropian y controlan el tiempo para la extracción máxima de la plusvalía. Pero el capital no quiere destruir el tiempo, porque crece al apropiarse del tiempo. De hecho, aun cuando quisiera, el capital no puede ni darse tiempo ni destruirlo. Esto significa que un principio irreductible de la esperanza mesiánica real es siempre estructural con respecto a la globalización capitalista. La persistencia del tiempo es infraestructural para el capital y no puede destruirse. En cuanto promulgación de la apertura de mundos por el venir del tiempo, la literatura mundial apunta a algo que siempre excede y desconcierta al capital. Propongo una explicación normativa de la literatura mundial, es decir, una que no analice simplemente cómo circulan las obras literarias a través del mundo o se producen con un mercado global en mente, sino que aspire a comprender la fuerza normativa que la literatura puede ejercer en el mundo, el horizonte ético-político que abre para el mundo existente. Una obra concreta de literatura mundial es una fuerza activa en la formación de mundos. Es el emplazamiento donde distintos procesos de worlding (mundialización) se juegan en un campo históricamente específico y un agente que participa e interviene en estos procesos ${ }^{4}$.

4. Worlding o, más habitualmente, worldling significa según el OED 'A cosmopolitan and sophisticated person'. Entiendo, sin embargo, que, en función de su argumentación, 
La literatura postcolonial es un caso ejemplar de literatura mundial en este sentido normativo fuerte, porque está implicada con la apertura de mundos y busca participar en procesos de mundanalidad. Es importante recordar aquí que la visión cartográfica del mundo está directamente relacionada con la historia del imperialismo europeo y su administración del tiempo de acuerdo con las coordenadas temporales de Greenwich. Los procesos cartográficos que crearon el sistema-mundo erradicaron otras culturas, sus formas de vida y sus temporalidades mediante su incorporación al sistema-mundo moderno, especialmente sus temporalidades, en las que las relaciones de la humanidad con los seres no-humanos no es de control instrumental y dominación. Estas tecnologías prosiguen de una forma todavía más intensa con la globalización capitalista. La desmundanización que producen incluye la desposesión colonial, la esclavitud de bienes, distintas formas de explotación capitalista y degradación medioambiental. La descolonización es precisamente un proyecto de re-mundanización de manera que puedan emerger nuevos sujetos en el mundo existente, transformándolo a través de esta emergencia. Esto implica el inicio de temporalidades nuevas o alternativas. Como parte de su vocación ético-política, las novelas postcoloniales que yo estudio proporcionan mapas cognitivos de la posición de las sociedades postcoloniales en las coordenadas globales de la administración del tiempo como, por ejemplo, los regímenes temporales del capital azucarero, el dinero turístico internacional, la ayuda humanitaria, los fondos de preservación de la vida salvaje o los fondos de desarrollo económico: Jamaica en las novelas protagonizadas por Clare Savage de Michelle Cliff, India en La marea hambrienta de Amitav Ghosh, Somalia en Regalos de Nuruddin Farah o Filipinas en State of War de Ninotchka Rosca o Halo Halo de Timothy Mo. Crean nuevas historias de pertenencia al mundo para personas del Sur postcolonial al recurrir a otras temporalidades que han persistido a partir de tradiciones precoloniales, no europeas; traen a un primer plano las tensiones con los regímenes capitalistas globales de gobernanza temporal y apuntan hacia la apertura de mundos.

Como indicación final, una advertencia: el concepto ontológico moderno de mundo que yo reconstruyo tiene una filiación específica con tradiciones religiosas abrahámicas, especialmente el cristianismo. A pesar de su tentativa de romper con los conceptos griego y cristiano de cosmos y mundo, la explicación fenomenológica de Heidegger sobre el mundo acaba

Cheah emplea worlding con el sentido de 'creación de mundos', que expreso en castellano con el verbo «mundializar» en una acepción distinta de la recogida por el DLE (actualización 2019) en cuanto 'Hacer que algo alcance una dimensión mundial'. [Nota del trad.] 
por afirmar una metafísica eurocéntrica de la presencia, porque privilegia el griego y el alemán como las únicas lenguas capaces de expresar el Ser. Idealmente debería haber situado el sistema-mundo capitalista moderno y la globalización en términos de complicidad entre eurocentrismo, colonialismo y modernidad y, después, re-conceptualizar el mundo a través de tradiciones culturales y religiosas no europeas como, por ejemplo, el concepto chino tianxia (天下, todo lo que está bajo el cielo), que no es shijie (世界), el título del famoso filme de 2004 de Jia Zhangke El mundo, o las distinciones en árabe, también en indonesio y malayo, entre alam, dunia y bumi -por ejemplo, el título del primer volumen de la "tetralogía de Buru", de Pramoedya Ananta Toer (1998, 2003, 2004 y 2006), es Bumi Manusia (Tierra humana)-, de forma que el sistema-mundo capitalista moderno pueda ser transformado para mejor a imagen de modernidades alternativas no occidentales y los ritmos de temporalidades culturales diferentes. Debería, de hecho, haber tantos conceptos de mundo como lenguas y culturas, y esto debería ser la única forma de resituar la literatura mundial. Pero una persona sola no puede hacer tanto; tiene que ser un trabajo colaborativo de investigadores de Europa, Asia oriental, el mundo árabe, África, etc.

Espero que esto pueda hacerse realidad; obviamente, es necesario algún tipo de apoyo institucional para que esta investigación comparativa de distintas ideas de mundo pueda comenzar, con la esperanza de que habrá de conducir a una explicación más plena de la literatura mundial. Gracias y aguardo la respuesta de David. [Aplausos]

DAVID DAMROSCH: Quiero traer muy decididamente a la atención de ustedes el libro de Pheng Cheah, así como el anterior, Spectral Nationality, que se publicó en 2003, el mismo año que mi What is World Literature?. Quiero plantear un par de preguntas generales, así como sobre la relación entre los estudios postcoloniales y los estudios de literatura mundial. El libro de Cheah tiene algunos elementos en común con otros libros recientes: en 2013 Against World Literature, de Emily Apter, y en 2015 Combined and Uneven Development: Towards a New Theory of World Literature, del Warwick Collective (WRec), justo un año antes del libro de Pheng. Considero que en estos tres libros hay cuatro dicotomías que se plantean de forma descarnada o cuyas relaciones no han sido suficientemente desarrolladas. La primera es la dicotomía entre tiempo e historia, particularmente historia literaria. La segunda es espacio frente a tiempo, y en especial la cuestión de lo cartográfico, esto es, si es o no demasiado sencillo decir que una cartografía espacial o física de la literatura mundial es meramente descriptiva, mientras que recartografiar temporalmente transforma realmente el mundo de una forma distinta. La tercera dicotomía es mundo frente a nación, en la que se supone que la literatura mundial no asume fronteras o incluso el desvanecimiento de 
las naciones, algo que no creo que haya sucedido. Finalmente, en un nivel político, la oposición entre revolución y reforma, o podría decirse radicalismo frente a liberalismo o progresismo, aunque creo que "progresividad» es un término que cualquiera de estas ramas puede adoptar.

Al pensar sobre temporalidad frente a historia, una de las cosas que realmente me ha sorprendido al leer recientemente estos tres libros de forma conjunta es que Emily Apter habla también de la necesidad de repensar las cartografías del tiempo, de la periodicidad. Habla sobre cómo se debe renunciar a una euro-cronología, de forma que ya toca algunos de los temas de Pheng, pero en el libro de Apter, así como en los otros dos, prácticamente todos los ejemplos son del siglo xx. Aunque indudablemente es importante repensar el modernismo o la modernidad, solo es la punta del iceberg de la periodización y, de hecho, hasta cierto punto el período menos problemático comparado con un término como medieval o clásico o antiguo, que no se ajustan tan bien a escala mundial como lo hacen las obras modernas. De hecho, muchos de los ejemplos de estos libros pertenecen a los últimos cincuenta años de la producción literaria, que supone el uno por ciento más reciente de la historia de la alfabetización hasta el momento y el siglo pasado solo el dos por ciento. En los Estados Unidos hemos tenido el movimiento "Occupy Wall Street» en virtud del 98 por ciento de la economía; creo que necesitamos ocupar la literatura comparada en virtud del 98 por ciento de la historia de la literatura, que se ve progresivamente obstruido.

Lo que quiero preguntarle a Pheng es esto: ¿cuál es el sacrificio de esa fuerte concentración en el presente si se quiere buscar temporalidades alternativas al tiempo que se obstruye la mayor parte de la historia de la literatura? Creo que tus últimos comentarios de hace un momento empiezan a responder mi pregunta, pero ninguna de las obras que discutes en tu libro son previas a 1900, y me pregunto si es necesario reducir tan dramáticamente la horquilla temporal para hablar de temporalidades alternativas. ¿Qué se gana con la atención por lo moderno? ¿Qué sacrificios ves en ese marco temporal?

PC: Diría en primer lugar que la forma en que se define la historia mundial, digamos por ejemplo por Hegel y, ciertamente, por Marx, es como un fenómeno moderno. Marx afirmó que la historia mundial solo tiene existencia con algo llamado mercado mundial. Es desde ese momento en adelante que se puede mirar atrás en la historia, incluido el pasado clásico, incluida la historia antigua, y decir que es parte de una teleología, una totalidad organizada que conduce a la modernidad. Por tanto, es una historia, pero una historia de la modernidad. En ese marco, se incluyen otras obras del pasado, de distintas épocas, como parte de la literatura mundial. 
Pero la literatura mundial es en realidad un fenómeno moderno. Estos textos antiguos son parte de una herencia que conduce a la modernidad. Con respecto a por qué escogí esos libros de literatura mundial en concreto en mi libro, hay una razón muy práctica.

Un comentario que algunos han hecho sobre mi libro -a diferencia del libro de David, que discute literatura no solo de distintos períodos sino en distintas lenguas- es que los textos primarios que examino, aparte de una serie de filósofos europeos, principalmente alemanes, son novelas recientes escritas en inglés. Cuando aún estaba con nosotros mi maestro Benedict Anderson, leía todo lo que yo escribía. Cuando le envié el manuscrito de este libro, lo primero que me dijo fue: «hablas de distintas temporalidades, pero ¿por qué todos los textos que discutes están en inglés?». Es solo por motivos prácticos. He dado charlas basadas en capítulos del libro, y nunca se me planteó ninguna pregunta sobre las novelas. Las personas del público me preguntan sobre teorías del mundo, porque no han leído las novelas pese a que estaba hablando de escritores bien conocidos como Amitav Ghosh y Nuruddin Farah. Había planeado escribir sobre un par de novelas indonesias, así como sobre Mo Yan, el novelista chino, pero entonces pensé que, si mi público no había leído Amitav Ghosh, entonces era aún más improbable que hubiese leído al novelista indonesio Eka Kurniawan, una de cuyas novelas no había sido traducida en ese momento. Por eso, por razones prácticas decidí escribir sobre textos que fueran más accesibles.

Se puede decir que esto también es parte del paradigma de la literatura mundial tal y como lo define David, que algo debe circular para tener algún tipo de impacto. Obviamente, las novelas que discuto en mi libro están escritas en el presente. Pero no tratan solo de fenómenos del presente. Se interesan por la historia del colonialismo europeo y su impacto en los mundos africano, caribeño y asiático e intentan resucitar temporalidades del pasado precolonial. Por ejemplo, The Hungry Tide (La marea hambrienta), de Amitav Ghosh, es una novela muy interesada por las tradiciones míticas sobre los espíritus de los tigres y las deidades de los bosques tanto en la tradición islámica como en el Asia del sur hindú. Nuruddin Farah hace uso de historias sobre jinns y espíritus de las tradiciones africanas. Por tanto, los propios escritores hacen referencias a otros lugares, otros períodos, otros mundos, pero, una vez más, de lo que se trata es de recuperar literatura y tradiciones orales del pasado para los propósitos de las sociedades africanas, asiáticas y caribeñas en el presente con el objeto de proyectar un futuro.

Si se piensa sobre la formación de la literatura mundial, una pregunta sencilla es: ¿para quién/qué es? Es para que la gente ahora dé sentido a qué es el mundo y qué es la literatura mundial, como parte de -para emplear una expresión pasada de moda- la "herencia humana", para una existencia 
en el presente y en el futuro. Es por eso que los escritos de la Antigüedad clásica, representados por ejemplo por Platón, Eurípides y otros, siempre se incluirán en la literatura mundial. Sin embargo, hay una distinción clara entre cómo se incluyen estas obras de las tradiciones clásicas en la literatura mundial y cómo se incluyen en investigaciones de orientación filológica, como los estudios regionales (Area Studies) o los estudios clásicos 5 . Por ejemplo, si se enseña y escribe sobre la literatura clásica india como un especialista regional, eso sería muy diferente de alguien que esté escribiendo y enseñando literatura clásica india como parte de la literatura mundial. Si se hace como parte de la literatura mundial, se estará enseñando y escribiendo muy probablemente en traducción y poniendo en contacto la obra en cuestión con textos diferentes de tradiciones y períodos históricos diversos para personas en el presente con el objetivo amplio de comprender el pasado y otras tradiciones culturales como parte de nuestro mundo común. Si se hace como parte de estudios regionales o de estudios clásicos, se buscará una inmersión completa en la tradición literaria clásica en cuestión o se imaginará penetrar en el mundo pasado. Creo que esa es la diferencia.

DD: Voy a seguir insistiendo en esto en la medida en que considero que implica aceptar una especie de teleología hegeliana hasta un extremo que no es realmente necesario. Aun cuando toda historia es hasta cierto punto una historia del presente, una cuestión distinta es tomar el pasado en sus propios términos y no simplemente por cómo ha sido reescrito en los últimos veinte o treinta años. Un pequeño ejemplo: el primer mecenas literario del mundo que se conoce es el Rey Šulgi de Ur, quien reinó en el siglo XXI antes de nuestra era, y en uno de sus poemas imagina su poesía formando parte de la literatura mundial, y ello en una época en la que nadie en el mundo escribía, con la excepción de Sumeria y Egipto. Dice:

Ahora, juro por Utu en este mismo día -y mis hermanos menores serán testigos de ello en las tierras lejanas donde los hijos de Sumer no son conocidos, donde la gente no tiene el uso de caminos pavimentados, donde no tienen ningún acceso a la palabra escrita- que el primogénito [en referencia a sí mismo] es un creador de palabras, un compositor de canciones, un compositor de palabras, y que ellos recitarán mis canciones como escritos celestes ${ }^{6}$.

5. Los estudios regionales de orientación filológica se corresponden con lo que en la Academia hispanohablante se denomina filologías nacionales. [Nota del trad.]

6. Este fragmento, que David Damrosch cita en la traducción al inglés del Electronic Text Corpus of Sumerian Literature de la Universidad de Oxford, pertenece al llamado Himno de Šulgi $B$, del que no tengo constancia que haya sido traducido al castellano en su totalidad. 
Así pues, el primer mecenas literario conocido utiliza la literatura para reforzar su imperio en la Mesopotamia meridional. Tenemos hoy la Epopeya de Gilgameš (Sanmartín 2010) porque este rey encargó poemas sobre Gilgameš. Šulgi fue un rey de la tercera dinastía de Ur; Gilgameš había reinado relativamente cerca, en Uruk. Šulgi refuerza su prestigio en otros poemas al hacerse pasar por hermanastro de Gilgameš y encarga baladas sobre Gilgameš (o Bilgames en sumerio) que se convertirán en el poema épico. Tenemos pues varias cosas que nos interesan hoy: el papel de la escritura para el imperio, el énfasis temporal en el futuro, pero se observará que no le interesa el capitalismo, que no existirá aún por varios milenios. En consecuencia, parte de mi pregunta acerca de este énfasis en tu libro, y en los libros de Apter y WRec, es que parece que la única clase de literatura mundial que merezca ser discutida es aquella que critica el capitalismo global, que a priori concierne solo a literatura muy reciente. Me preocupa que obstruir todas las temporalidades previas signifique, de hecho, renunciar a favor de la escala temporal del capitalismo global hasta un grado innecesario.

Esta pregunta tal vez también tenga que ver con la cartografía del espacio, con la pretensión de que dichos mapas solo describen el mundo, mientras que las cartografías de la temporalidad lo transforman. A este respecto quiero indicar que no hay una única cartografía de la literatura mundial. Existen versiones distintas en diferentes partes, y no solo en los Estados Unidos. Así, como tú mismo dices, necesitamos ver mundos distintos en lugares diferentes. [Muestra una diapositiva] Aquí hay un par de estudios alemanes recientes que son muy distintos. Hay el tipo de ideal ilustrado en el manual de 2015 de Dieter Lamping con ensayos concisos sobre escritores mundiales Meilensteine der Weltliteratur. Von der Aufklärung bis in die Gegenwart (Hitos de la literatura mundial desde la Ilustración hasta el presente). Se apreciará que en la ilustración de la tapa la literatura mundial vuela por encima de las nubes, todo muy trascendente. Y un año antes tenemos a Sigrid Löffler, una personalidad de la televisión y los medios, con Die neue Weltliteratur und ibre großen Erzäbler (La nueva literatura mundial y sus grandes narradores). Es otra vez un panorama de "grandes obras", pero las grandes obras son en esta ocasión novelas de escritores migrantes, muy contemporáneas. La tapa muestra a un librero en las calles de Calcuta, y no estantes de libros que se remontan hasta las nubes. Son dos clases de

\footnotetext{
Reproduzco aquí dicho pasaje en traducción de Escobar OrTiz $(2017,65)$. Para una traducción al castellano del Himno de Šulgi A, remito a Lara PEINADO (1988, 162-167). En relación con la argumentación de Damrosch, también es oportuno señalar que en el himno B, así como en el himno C, se presenta al Rey Šulgi como un políglota, conocedor de sumerio, elamita, amorita, subario y acadio o meluhhano; véase RuBIo (1999). [Nota del trad.]
} 
literatura mundial, y ambas tienen un papel hoy, no una más que la otra; de hecho, una está imbricada en elementos de la otra y viceversa.

PC: ¿Podemos volver a la anterior diapositiva [la del Rey Šulgi]? Cuando dice "mis canciones como escritos celestes", no dice escrituras mundiales/ mundanales. La pregunta es (y no conozco estas lenguas): ¿qué significa cosmológicamente "cielo» en relación con la Tierra? ¿Significa lo que habitualmente entendemos por mundo? Lo que quiero decir es que literatura mundial depende de una definición moderna de mundo. Además, no es totalmente exacto decir que mi posición sobre la literatura mundial excluye la literatura del pasado. Aunque discuta primariamente obras literarias recientes, la literatura del pasado también mundializó un mundo pasado. Para el Rey Šulgi, la literatura tenía ese poder de mundialización. Escritores y lectores de hoy pueden aprovechar literaturas de períodos previos para re-mundializar el mundo presente frente a la globalización capitalista. Como discuto en mi libro, esto supone intentar perturbar el tiempo vacío homogéneo del capitalismo global al invocar otras temporalidades.

DD: Pero yo estoy diciendo que esto significa algo distinto, y tenemos una comprensión amplia de las temporalidades del mundo si imaginamos -como lo hicieron los sumerios- que la realeza procede del cielo, es un don de los dioses; el rey representa el punto de encuentro entre lo celestial y lo terrestre. Cuando vislumbra que su fama se extenderá por el mundo gracias a sus poemas, es el mundo real lo que él está previendo. En consecuencia, y para volver a la pregunta sobre las cartografías, esto conduce a la cuestión de revolución frente a reforma, o lo radical frente a lo progresista. Pienso en las cartografías radicales de los realistas. Aquí quisiera hacer referencia a la gran investigadora del surrealismo Anna Balakian. Fue una exiliada armenia cuya familia abandonó Turquía tras el genocidio y acabó en los Estados Unidos. En un ensayo tardío afirma que en la universidad

[i]ngenuamente y quizás con el idealismo de la juventud, pensé en la literatura comparada como un antídoto frente al nacionalismo exacerbado, y el surrealismo era la única literatura que estaba reaccionando frente a las divisiones nacionales, incluso traspasando las fronteras entre las artes. Pensé, inocentemente, que con las perspectivas de la literatura comparada y la diseminación de los principios del surrealismo podríamos cambiar el mundo. (Balakian 1994, 77)

No quiere describir el mundo, sino que quiere cambiarlo; y añade: «Así que compré un billete e hice una pequeña inversión financiera que determinó el resto de mi vida» (Balakian 1994, 78). 
Era un billete para viajar al segundo encuentro de la Asociación Internacional de Literatura Comparada en Chapel Hill, Carolina del Norte en 19587. Como ilustración del tipo de cartografía por el que estaba interesada, he seleccionado este gran mapa [muestra la diapositiva] del libro de Delia Ungureanu $(2017,180)$ From Paris to Tlön: Surrealism as World Literature. Tenemos a André Breton y sus amigos que dibujan un mapa que muestra Le monde au temps des Surréalistes, el mundo en la época de los surrealistas. Como se lee en el libro de Delia, Breton afirma que la cartografía de meridianos, paralelos y latitudes encierra el mundo en una jaula, y los surrealistas van a hacer explotar ese gráfico. Así que, en vez del ecuador habitual, tenemos una línea ondulada, y el Pacífico como el centro del mundo. Inglaterra ha desaparecido prácticamente, Irlanda es la isla grande a la izquierda, con una Inglaterra diminuta dibujada como el ojo de una cerradura, y tenemos a París, pero no mucho de Europa. Rusia y China son enormes; tristemente, no hay rastro de Japón, pero están las Filipinas, Indonesia y el océano Índico. Se muestra L'ile de Pâques, la Isla de Pascua, porque Breton estaba recopilando obras de arte de allí. No está Estados Unidos, pero sí Alaska, Labrador; México también está ahí, y una especie de minúscula Sudamérica. Es un mapa que está cambiando el mundo de una forma muy explícita con una descripción cartográfica diferente. Es por eso por lo que me pregunto si es necesario decir que es el mapa temporal el que cambia el mundo y pensar sobre la circulación y el mapa espacial del mundo como algo conservador, el statu quo. No creo que sea lo que pensaban los surrealistas, o lo que estamos haciendo hoy.

PC: Has comenzado con la dicotomía de temporalidad frente a historia y, bajo mi punto de vista, la historia es una categoría temporal. Por lo tanto, la historia no se opone a lo temporal. La dicotomía es espacio frente a tiempo. Pienso que incluso cuando se habla del tiempo, por ejemplo, el hecho de que las zonas horarias estén determinadas por meridianos y paralelos es en esencia un sentido espacializado del tiempo. Lo que me interesa es comprender el mundo en un sentido auténticamente temporal.

7. En este II Congreso de la AILC/ICLA, Balakian presentó una ponencia titulada «The Objective of Comparative Literature» (El objetivo de la literatura comparada) en el marco de un simposio sobre la enseñanza de la disciplina. En ella defendía que la literatura comparada "interesará no solo al especialista o estudiante de doctorado ya formado en los hábitos de una investigación unilateral, sino también al estudiante no especializado -tanto de grado como de postgrado- como parte de su desarrollo cultural general» (Balakian 1959, 237). [Nota del trad.] 
La pregunta es -incluso si haces este tipo de cosas [apuntando hacia el mapa surrealista], sigue siendo un tipo de mapa- ¿cuál es el fundamento para esta reevaluación y transformación de una cartografía dada? Se puede comprender esto en términos de personas imaginando un mundo que es distinto, entonces re-cartografías el mundo de una forma distinta tal y como lo hacen los surrealistas. Pero la pregunta es: ¿̇cuál es el impulso detrás de este mapa? ¿De dónde procede el principio de novedad? Me parece que uno siempre debe volver a la pregunta acerca del tiempo, porque es con el darse y el venir del tiempo que algo nuevo emerge y el mundo se reabre nuevamente. El tiempo del mundo en este sentido no es el tiempo de las historias literarias globales.

DD: Pero se le puede dar la vuelta a esa pregunta. Tenemos cosas de la Isla de Pascua, manifestaciones artísticas nunca vistas antes, tenemos cosas de Irlanda, que para Breton es más importante que Inglaterra como lugar de un arte revolucionario. Como se puede decir también de Pascale Casanova, su mapa es pro-periferia, la periferia como el motor del desarrollo literario. No se trata de la opción o esto o lo otro; lo espacial puede ser tan revolucionario o progresista como lo temporal.

PC: Si piensas sobre la circulación, y desde luego no la estoy descontando, creo que hay un elemento importante de desacuerdo entre nosotros. Dices que tengo una concepción limitada sobre la circulación, que no considero que la circulación pueda tener efectos "progresistas" o radicales. Pero no es así. Cuando pensamos acerca del trabajo de alguien como Paul Gilroy cuando examina cómo ha sido utilizada la Biblia por los escritores y pensadores afroamericanos para comprender la libertad desde la esclavitud, entonces está claro que la circulación de textos puede tener efectos progresistas. La pregunta central es básicamente una pregunta ontológica: ¿qué hace que la circulación tenga lugar? Solo se puede hacer circular cosas entre sujetos; y solo se puede hacer circular cosas si hay tiempo, si el tiempo persiste. Hace falta espacio, pero para que algo se mueva, el tiempo debe persistir. No hay circulación, no hay movimiento sin tiempo. Por tanto, ontológicamente, el tiempo es la categoría primaria, y es de ahí de donde proceden los efectos progresistas de la circulación.

Otra cuestión que debo aclarar es que, debido en parte a mi interés por la globalización, la circulación de los bienes está siempre ligada de una forma u otra a la explotación capitalista. Ese es el aspecto que señalabas al mencionar todos esos libros de Apter, el wrec; estamos identificando circulación con explotación capitalista. Sin embargo, hay explicaciones de los efectos progresistas de la circulación de bienes. Si piensas en mi maestro, en el libro de Ben Anderson Imagined Communities (Comunidades 
imaginadas), que tanta influencia ha tenido en la teoría y la crítica literaria, él emplea la expresión capitalismo impreso. El capitalismo impreso produce algo que es progresista. Es una de las condiciones materiales fundamentales de la nación como comunidad imaginada; Anderson considera la nación como una comunidad popular caracterizada por la igualdad y libertad soberana. Pero, bajo mi punto de vista, el vínculo con el capitalismo siempre va a estar ahí con la circulación de bienes; por tanto, no es una cuestión de decir simplemente que la circulación como tal es liberadora, sino que más bien se trata de analizar este o ese caso particular cuando la circulación conduce a algo que es progresista. Haces otra distinción entre revolución y reforma, que se corresponde con radicalismo frente a liberalismo. Tal vez sea un error en mi interpretación, pero cuando sostienes que hay un incremento semántico cuando algo circula ${ }^{8}$, inmediatamente me viene a la mente el capítulo cuarto del primer volumen de El capital, en el que Marx describe la auto-valorización del capital; dice «dinero que incuba dineron?. Solo por circular, el capital se multiplica infinitamente, de manera que el dinero incuba dinero. Pareciera que fuera un poco así, que algo, solo por su movimiento, se incrementa y se aumenta a sí mismo casi exponencialmente.

DD: Déjame hacer un comentario sobre eso, en parte porque esos pasajes que tienes en mente de mi What Is World Literature? los escribí hace quince años y he intentado pensar más sobre eso desde entonces. En aquel entonces la circulación me pareció un buen punto de partida, porque, como dijiste, no sería posible enseñar Amitav Ghosh o Nuruddin Farah a menos que estuvieran disponibles en el mercado para ser asignados a los estudiantes, sea en papel o en versión electrónica. Incluso los clásicos antiguos pueden tener efectos progresistas. Ngũgĩ wa Thiong'o (2018) tiene unas memorias sobre sus dias de escuela, In the House of the Interpreter (En la casa del intérprete), en las que dice que sus profesores del instituto de secundaria hacían que leyeran Shakespeare para convertir a los niños en buenos ciudadanos, pero dice que él y sus amigos montaban

8. Hace referencia aquí Cheah a una de las definiciones de literatura mundial por parte de DAmrosch $(2003,281)$ : «La literatura mundial es escritura que gana en traducción». [Nota del trad.]

9. «El valor, pues, se vuelve valor en proceso, dinero en proceso, y en ese carácter, capital. Proviene de la circulación, retorna a ella, se conserva y multiplica en ella, regresa de ella acrecentando y reanuda una y otra vez, siempre, el mismo ciclo. D-D', dinero que incuba dinero -money which begets money-, reza la definición del capital en boca de sus primeros intérpretes, los mercantilistas» (MARX 2008, 189). 
producciones de Enrique IV o El rey Lear y se daban cuenta: «Vaya, puedes destronar a un rey». Y he estado también pensado sobre algo que Wiebke Denecke (2013) dice en su libro Classical World Literatures: Sino-Japanese and Greco-roman Comparisons: los modelos de circulación no son tan interesantes para el mundo antiguo porque muchas cosas no circularon. Si se piensa en la literatura mundial antigua, se van a comparar obras de culturas que eran muy distintas. Y ella hace un trabajo muy bueno al respecto. Pero también he estado reflexionando acerca del mundo en la nación y estoy totalmente de acuerdo con que un buen estudio de literatura mundial no debería asumir que las fronteras se han diluido o que la nación ha dejado de ser una entidad significativa. Todo lo contrario.

Esto es verdad incluso en el caso de grandes naciones, y lo vemos particularmente en los países pequeños. Tomemos como ejemplo al poeta nacional esloveno France Prešeren, cuyo poema "Zdravljica» (Un brindis) se ha convertido en el himno nacional:

Dios Bendiga a todas las naciones,

Que dedican tiempo y trabajo para ver el día brillar,

Cuando en la tierra, ninguna guerra,

Ninguna contienda celebrará su dominio,

Durante mucho tiempo,

para ver a todos los hombres libres,

¡No habrá más enemigos, que todos vecinos sean! $!^{10}$

Este himno internacionalista es el himno nacional de Eslovenia, pero no lo era cuando Prešeren falleció en 1849. Es el himno nacional solo desde 1989, en el momento previo a que Eslovenia se convirtiera en una nación independiente un par de años más tarde. Cada vez pienso más y más sobre esta clase de inversión entre fondo y figura, que son mundo y nación. Solía pensar «aquí está la nación y aquí, fuera, está el mundo», pero, si uno lo piensa en términos de mercado, no como una metáfora sino como una realidad, el mundo solo existe dentro de un mercado nacional, en un contexto nacional, en una cultura nacional, sea progresista, regresivo o lo que sea.

10. Es la séptima estrofa de este poema la que se ha convertido en el himno nacional esloveno. Damrosch la cita en traducción al inglés, que aquí reemplazo por la traducción al castellano ofrecida por la Asociación Cultural Esloveno-Venezolana; <http://culturaeslovena.blogspot.com/2017/01/el-himno-nacional-de-eslovenia.html> (Noviembre de 2019). [Nota del trad.] 
Aquí va mi pregunta final para proseguir un poco más con el tema de revolución frente a reforma. Me parece que en libros como el de Apter o el Wrec $-\mathrm{y}$, probablemente, también en ocasiones el tuyo- el foco se pone de una forma tan fuerte en obras que critican el capitalismo global que el resultado es un filtro muy particular de la literatura mundial que se produce hoy día, incluso en el Sur Global. Estaba pensando sobre esto en relación con las Comunidades imaginadas de Anderson. Varias veces él menciona a Pramoedya Ananta Toer, sobre quien tú has escrito mucho, y Anderson escribió también mucho sobre Pramoedya, pero también era un gran especialista en la literatura tailandesa y compiló todo un volumen de traducción de relatos tailandeses, pero nunca habló sobre Kukrit Pramoj. Me sorprende que Kukrit nunca aparezca en la obra de Anderson o en la obra de cualquier otro que conozca. ¿Qué significaría incorporar Kukrit a Pramoedya? Ambos son autores de novelas destacables sobre cuatro generaciones acerca de la modernidad y el advenimiento de su nación, desde el inicio del siglo Xx en adelante. Has escrito con brillantez sobre la "tetralogía de Buru» en tu libro Spectral Nationality. Pero el libro de Kukrit Si Phaendin (Los cuatro reinos) precedió en una década o así a la "tetralogía de Buru» de Pramoedya. Estos reinos comienzan en la década de 1890 con la vida bajo el reformista Rama V y luego sus tres sucesores hasta el final de la Segunda Guerra Mundial y el periodo de guerra durante la ocupación japonesa. La historia se cuenta a través de una heroína, Mae Phloi, quien va a la corte de niña y vive a lo largo de esas generaciones. Es un libro extenso, pero es una historia en cuatro ciclos y se publicó unos cuantos años antes que Pramoedya comenzara a trabajar en la «tetralogía de Buru». Kukrit es un tipo realmente interesante. Comienza como un escritor anticolonial; escribió su primer libro, A King of Siam Speaks (Habla un Rey de Siam), junto a su hermano en un esfuerzo por restaurar la historicidad frente al modernizador Rama IV, quien se había convertido en el modelo del rey autocrático, atado a la tradición, del filme hollywoodiense Anna y el rey. Kukrit y su hermano estaban tan molestos con esta película que escribieron este libro cuando aún tenían veinte años con el objeto de corregir la información para los lectores en inglés. No mucho después, Kukrit funda un periódico -perfecto para Benedict Anderson- titulado Siam Rath (La Nación Tailandesa). También se convierte en actor, funda un grupo de danza clásica tailandesa y escribe una adaptación teatral de Rashōmon (basada en el filme de Kurosawa), en la que hace uno de los papeles protagonistas. Mientras tanto funda también un partido político y utiliza su periódico para comentarios y reportajes tanto políticos como culturales. En 1962 protagoniza con Marlon Brando un filme titulado Su Excelencia el embajador, en el que retrata al primer ministro de un país del Sudeste asiático que tiene 
PHENG CHEAH Y DAVID DAMROSCH

que habérselas con la implicación estadounidense en Indochina. Diez años más tarde se convierte en el primer ministro de Tailandia. ¡Uno no puede inventarse todo esto!

Y así es que me pregunté: ¿por qué nunca se habla de él? Su vida y su obra tan poliédrica afectan a muchas cosas que interesan a la gente. Las únicas diferencias en las que puedo pensar acerca de por qué Anderson escribió sobre Pramoedya pero nunca sobre Kukrit -aunque conocía perfectamente tanto la política y la cultura tailandesa como lo relativo a Indonesia- son dos. La primera es que Kukrit no era de izquierdas, sino un monárquico. La segunda es que no era laico, sino budista. Su otro mejor libro disponible en inglés es Many Lives (Muchas vidas), en el que presenta qué sucede cuando un ferry se hunde en el río Chao Phraya, que atraviesa Bangkok, y la mayoría de los pasajeros se ahoga. Proporciona una serie de historias que retratan cómo el karma de cada uno los condujo a ese punto. Es un libro muy bonito, profundamente budista, como también hay un subtexto budista en Si Phaendin. Junto a su perspectiva budista, políticamente Kukrit es una especie de conservador progresista, si se puede decir así. Es un modernizador activo y tiene un fuerte interés en la cultura femenina; crea una heroína fuerte a la que le otorga más protagonismo que cualquiera de las mujeres en la "tetralogía de Buru». Debería estar en el centro de lo que pensamos que acontece en el Sur Global, pero nunca se discute sobre él.

Creo que, en términos de cartografías de la circulación, todos quienes estamos interesados en la literatura mundial somos activistas dentro de nuestros propios contextos, y eso es realmente importante para abrirse a un repertorio literario más amplio, con mayor cabida, sea en el Sur Global, sea en el Sudeste asiático, sea con la política de la narración en términos generales. Se han escrito sobre Pramoedya alrededor de cuarenta y cinco estudios de acuerdo con las fuentes proporcionadas por la Bibliografía Internacional de la Modern Language Association of America. Sobre Kukrit, un único artículo, un panorama biográfico de cinco páginas. Por eso me parece que solo hemos comenzado a hacer lo que debemos hacer en cuanto investigadores y activistas de la literatura mundial, que es abrir el mundo al elenco de escritores que han sido relegados por muy distintas razones, incluyendo los filtros que, en general, los estudios postcoloniales han impuesto en el archivo al que desean mirar.

PC: Estoy de acuerdo sobre la expansión del repertorio de textos, pero volvamos un poco atrás para hablar sobre la dicotomía entre mundo y nación. No creo que estemos en desacuerdo a este respecto. Porque para que cada nación se establezca como tal y sea respetada, debe haber una estructura internacional de reconocimiento. Tiene que ser reconocida por 
otras naciones, y la esfera cultural es importante para ello. Este fue, por ejemplo, un factor importante de estímulo en el Clasicismo de Weimar y las revoluciones de 1848 basadas en las lenguas vernáculas. Toda constitución socialista posee una retórica internacionalista. La gente se olvida de que el socialismo existente en la actualidad no fue realmente cosmopolita, sino fundamentalmente internacionalista. Por ello, en este sentido, se es primero miembro de una nación y luego, a través de la nación, miembro de la humanidad. Creo que es un dictamen muy importante para Goethe.

DD: Y el poema de Prešeren que cité tiene mucho que ver con las naciones juntas.

PC: En relación con por qué tan pocas personas han escrito sobre Kukrit en el marco de los estudios literarios postcoloniales del Sudeste asiático, obviamente algunos escritores y teóricos tienen una preferencia por una agenda más radical. Diría esto de Ben Anderson y también de mí mismo. Pero todo el mundo tiene este tipo de sesgos, se admita o no.

Bajo mi punto de vista, una re-mundialización más radical del mundo tiene lugar a través de la tradición revolucionaria. Pero la revolución no siempre es posible. Diría incluso que hoy día la revolución ya no es posible, en cuyo caso otros tipos de escritos serían importantes. Con respecto a los sentimientos monárquicos de Kukrit, Ben Anderson estaba escribiendo antes de fallecer un libro sobre monarquías, por las que sentía un profundo desagrado debido a su bagaje político. Pienso en consecuencia que debió apartarse de Kukrit por su política, pero no con seguridad por ser budista. El budismo del Sudeste asiático, especialmente en Tailandia, es maravillosamente extravagante. Hay intertextualidad budista en la brillante película de Apichatpong Weerasethakul Tropical Malady (Enfermedad tropical), de 2004, por ejemplo. Ben escribió en 2012 un libro fascinante en el que exploraba aspectos del budismo tailandés titulado The Fate of Rural Hell: Ascetism and Desire in Buddhist Thailand (El destino del infierno rural: ascetismo y deseo en la Tailandia budista).

La otra cuestión que planteas tiene que ver con la traducción, los mercados y los bienes; y mencionaste a Ngũgĩ. Comenzó a escribir como James Ngũgĩ. Más tarde, en determinado momento, decidió que no quería soportar más el yugo del colonialismo y se deshizo del «James». Después, comenzó a escribir solamente en kikuyu. Pero una vez que escribía solo en kikuyu, sus libros fueron censurados y prohibidos en Kenia, y solo pudo sobrevivir en traducción al inglés. Es por eso que creo que cuando se trasladó desde la Universidad de Nueva York a la de California Irvine fundó el Centro de Escritura y Traducción. Se interesó mucho por la traducción y el tipo de cosas que mencionaste, cómo Enrique IV tiene que ver con 
el regicidio, etc. Pero, al mismo tiempo, recordarás el famoso ejemplo de Writers in Politics (wa Thiong'o 1997) sobre el papel de la literatura inglesa con respecto a la imposición de valores eurocéntricos, cuando Ngũgĩ habla sobre su hijo, quien nunca había visto un narciso y, al memorizar el poema de Wordsworth «I Wandered Lonely as a Cloud» (Erraba solitario como una nube), piensa que el narciso es un tipo de pez. O, cuando vives en África, ¿cómo puedes identificarte con las imágenes poéticas sobre el invierno, el otoño, la primavera? Para Ngũgĩ, la circulación institucionalizada de la literatura europea mediante la educación colonial representaba una especie de lavado de cerebro con efectos muy negativos. Así que, por una parte, la traducción y la circulación de textos pueden funcionar de una forma positiva. Pero, por otra parte, pueden tener también efectos alienantes y violentos. Si piensas en ello, la enseñanza colonial de literatura europea como parte del currículo escolar en los países colonizados, ese fue quizás uno de los primeros ejemplos de literatura mundial institucionalizada, ¿no te parece? De forma que la literatura mundial ha sido históricamente utilizada para fines coloniales. Tu antiguo colega de Columbia, Gauri Viswanathan, escribió sobre esto en Masks of Conquests (Máscaras de las conquistas). Por eso creo que no debe perderse de vista.

Por otra parte, y a diferencia de Emily Apter o Aamir Mufti, no estoy en contra de la traducción y la escritura anglófona. Y no estoy en contra de los mercados. Por ejemplo, el escritor indonesio Eka Kurniawan escribió una novela titulada Lelaki Harimau (Hombre Tigre) que quería discutir en What Is a World? Pero no lo hice porque elegí escribir sobre obras que pudiesen ser leídas en inglés, y no se había traducido aún cuando estaba escribiendo mi libro. Después la novela se tradujo como Man Tiger y hace poco publiqué un artículo sobre ella en Diacritics (Cheah 2017) ${ }^{11}$. La razón por la que se tradujo es porque Ben presionó a la editorial Verso para que la tradujera. Ben mismo fue un traductor prolífico de literatura tailandesa, indonesia y tagalo. Así que pienso que la traducción y la circulación son muy importantes. Otro ejemplo: estaba cenando hace muchos años en Macao con la escritora taiwanesa Li Ang y Howard Goldblatt, un traductor muy conocido de literatura china. Estábamos hablando sobre cómo probablemente Mo Yan no hubiese recibido el Premio Nobel si no hubiera un traductor tan capacitado como Howard, quien tradujo su obra. Más tarde, tu colega David Wang dijo lo mismo en una conversación privada, que Mo Yan debería estarle agradecido a Howard. Y esto también se aplica al caso

11. Mantengo el título en inglés al ser la traducción a la que hace referencia Pheng Cheah. [Nota del trad.] 
de Ngũgĩ; obviamente la traducción es muy importante porque si su obra en kikuyu no se hubiese traducido, no habría sobrevivido.

En el caso de la "tetralogía de Buru» hay una escena muy interesante justo al final de la cuarta novela, Rumah Kaca (La casa de cristal), donde vemos que las primeras tres novelas son en origen una serie de libretas escritas por un pionero de la revolución de las Indias Orientales. Pero el revolucionario es encarcelado y sabemos que tendrá un mal final. La única forma de que los libros sobrevivan y se conviertan en los primeros tres libros de la tetralogía es dárselos a alguien en quien se pueda confiar que proseguirá con la vocación revolucionaria, pero esa persona ha emigrado a Francia. Y por ello se habla de la "casa temporal", el espíritu de la nación indonesia que espera a ser repatriado. De forma que uno tiene que situar en cada caso los efectos de la traducción y la circulación, y hay ejemplos en los que la circulación tiene efectos nocivos.

DD: Y, desde luego, la «tetralogía de Buru» ha sido prohibida en Indonesia, y el traductor, que es un diplomático australiano, ha sido expulsado del país. Así que es complicado.

PC: Traducción, circulación, son cuestiones importantes. Pero, bajo mi punto de vista, la asociación de la circulación de bienes con el capitalismo, eso no puede perderse de vista. Además, también es importante tomar en consideración esos efectos sobre los que Aamir Mufti (2018) escribe en su libro, que la literatura mundial anglófona distrae la atención de la literatura en otros idiomas. Así que pienso que cualquier noción desarrollada de literatura mundial necesita replantearse conceptos de mundo. Entonces tienes que tomar en cuenta cuáles son esos distintos conceptos de mundo en lenguas diversas. Por ejemplo, en francés no hay diferencia entre "el mundo" y «el globo»: se utiliza mondialisation tanto para mundialización como para globalización. Así que es importante resituar las ideas sobre la literatura mundial en conceptos distintos de mundo de distintas lenguas. Y después, para comprender estos conceptos de mundo en términos de su encaje en un contexto más amplio, tiene que haber algún tipo de actuación con los estudios regionales, y no solo un acercamiento casual.

La traducción es importante, pero al mismo tiempo y siendo avaricioso, quiero que se dé en ambas direcciones, con un vínculo con un conocimiento profundo de otras regiones mundiales. Pienso, finalmente, que es aquí donde mi teoría de la literatura mundial difiere de la tuya. Para mí, el mundo es la apertura mediante la cual un individuo o un colectivo emerge y llega a ser en un campo inmanente de fuerzas políticas, económicas, sociales y culturales, y la literatura mundial es la literatura que mundializa, practica esa apertura. Así que miro qué hace una obra literaria dada en un 
campo de fuerzas, y esto significa mirar a la interacción de la literatura con las fuerzas y los procesos no literarios. Mientras que, en tu caso, pienso que la literatura mundial implica las conexiones extensivas y expansivas que las obras literarias elaboran a través de la circulación global, y esto implica que tu enfoque es más historiográfico-literario. Pero no son enfoques mutuamente excluyentes. Por ejemplo, yo también tomo en consideración la influencia literaria y la intertextualidad cuando examino la fuerza de mundialización de una obra literaria, por ejemplo, cómo Amitav Ghosh depende de las Elegías de Duino, de Rilke, en La marea hambrienta. Pero hay una diferencia en el centro de atención. Esto probablemente se debe a que tú enseñas en un departamento de literatura, mientras que yo no lo hago desde que me trasladé a Berkeley en 1999.

DD: Confío en que todo el mundo esté encontrando esta discusión interesante. Pienso que puede ser un buen momento para dar la palabra al público para preguntas, comentarios, objeciones. Veamos hacia dónde quieren ir desde aquí.

PREGUNTA: Gracias por sus presentaciones, fueron fantásticas. Solo quería preguntar acerca de Heidegger, dado que habló mucho sobre él y las temporalidades. Cuando Heidegger habla sobre temporalidades en ¿Qué es metafisica?, discute el concepto de tedio y que las cosas tienen su tiempo propio, y si las encuentras a destiempo, entonces esto provoca tedio, es decir, una potencialidad que se pierde. Me preguntaba si en el contexto de la literatura esto significa que, además de literaturas que son apropiadas para recuperarlas ahora, hay literaturas que debemos dejar morir, literaturas con las que estamos a destiempo, literaturas que necesitan verse en su finitud y decir, «vale, no tenemos tiempo para ti, o tu momento volverá más tarde otra vez».

PC: Es una muy buena pregunta. Desde luego, la pregunta más amplia debería ser si es posible separar la explicación del mundo y del tiempo por parte de Heidegger de su antisemitismo. Porque, obviamente, consideró muy seriamente que las únicas dos lenguas en las que se podía pensar filosóficamente eran alemán y griego debido al estatuto de la flexión y la fusión en estas lenguas. Alguien en el seminario me preguntó antes qué había de los Cuadernos negros. El concepto de mundo recorre toda su obra, y en el medio de ella está ese pasaje famoso que aprovechó Agamben en el que dice que el ser humano tiene mundo y es formador de mundo (weltbildend), mientras que el animal es pobre en mundo, weltarm, y la piedra carece de mundo, weltlos, pero desde luego asumiendo implícitamente que, en la medida que eres un Dasein humano, un mundo siempre co-pertenece y te pertenece. Pero entonces en los Cuadernos negros sostiene que los 
judíos carecen de mundo, lo que obviamente es muy perturbador. Aquello que él privilegiaba no era la literatura per se, sino Dichtung, poesía, y más específicamente dos poetas, Hölderlin y Rilke. Así que obviamente no se puede tomar todo Heidegger, porque diría que hay literaturas que no cuentan, que no expresan lo que él llamaría una "auténtica temporalidad» y deben ser descartadas.

PREgunTA: Su explicación de la literatura mundial se enmarca en una defensa en contra de la relegación de la literatura a manos de una visión marxista de la cultura. Mi pregunta es: en su análisis usted aún le otorga al capital una importancia analítica central, ¿por qué?

PC: Debo decir que eso es en parte porque hoy estamos discutiendo el libro de David junto a los de Pascale Casanova y Franco Moretti, y pienso que la diferencia con el libro de David radica en que de hecho él realmente valora leer literatura, y una parte importante acerca de la literatura mundial es que debe ser literatura. No hay eso en Franco Moretti. Para Franco, no hay diferencia entre un tebeo de quiosco y una obra que pueda ser considerada "literatura» en el sentido culto. Para él, está muy claro que se trata de la circulación por la mera circulación. Es aquello que vende más, aquello que es el bien más valorado, eso es lo importante. Y si se revisa el libro de Casanova, que no es pequeño, no hay lectura directa (close reading). Uno hasta sospecha que no leyó todos estos títulos que menciona en su libro. Así que fue en contra de ese tipo de reduccionismo que insisto en la especificidad de la literatura y en el vínculo entre literatura y mundo. Por otra parte, hay cierto juego de prestidigitación, porque la primera parte de mi libro trata de filosofías del mundo, lo que conduce a la idea de mundialización y el nexo entre mundo y literatura, pero después, cuando hablo sobre ejemplos de literatura mundial, estudio textos literarios que se sitúan en contra de la globalización capitalista. Ese es mi propio sesgo político, y también porque me interesan las cuestiones de la globalización. No se puede entender en términos marxistas la fuerza de mundialización en la que se sustenta este tipo de literatura mundial postcolonial. La pregunta es si mi explicación normativa de la literatura mundial funciona o no con textos que no tienen que ver con la globalización postcolonial, que no son literatura postcolonial, y mi respuesta es: díganme ustedes si creen que la teoría es aplicable. Mi obra es en primera instancia un texto de teoría literaria. No enseño en un departamento de literatura, así que no practico la historia literaria o la historiografía literaria. De manera que la pregunta es si esta explicación de la literatura mundial resulta interesante, si es plausible en el caso de que no se esté estudiando literatura postcolonial. Puede que no sea interesante, y no pasa nada. 
DD: Mi punto de vista es que es más interesante cuando una lectura tiene que adaptarse a un archivo más amplio. Tomemos el ejemplo de la "tetralogía de Buru» de Pramoedya. El volumen cuarto es narrado por un oficial de policía que ha estado destruyendo al héroe, de forma que se nos da la historia a través de alguien que está profundamente investido del statu quo. Es realmente interesante que este volumen sea una reelaboración brillante de Max Havelaar o las subastas de café de la Compañia Comercial Holandesa, de Multatuli, cuyo narrador es un idiota llamado Droogstoppel ('Rastrojo seco'), quien consigue los papeles de este oficial colonial que ha estado intentando trabajar contra los excesos del gobierno holandés en las Indias Orientales. Este narrador idiota, brillantemente cómico, describe acontecimientos horribles que no comprende, y Pramoedya rehace esto de una forma oscura, absolutamente sorprendente. Es una clase distinta de enfoque para leer a Pramoedya a través de unas lentes histórico-literarias, pero esas lentes lo sitúan tanto en términos literarios como en términos de la lucha anticolonial más amplia. Multatuli no es exactamente un anticolonial; como Kipling, discute en contra de los excesos del sistema, era un reformista. Y su libro tuvo en efecto real en la vida que el de Pramoedya no tuvo.

PC: No, pienso que es de absoluta importancia, pero para comprender esto, se necesitaría establecer conexiones con los estudios regionales, mirar la historia colonial. Es necesario hacer esto para ser capaz de entender qué era el Cultuurstelsel (Sistema de Cultivo) y la Ethische Politiek (Política Ética), etc. Si coges las novelas en un departamento de literatura en el que probablemente se leerá a Pramoedya junto a Salman Rushdie o quien sea, entonces ese es el material que no vas a entender. En cierto momento después de acabar de escribir sobre la "tetralogía de Buru", pensé en escribir sobre la maravillosa épica marítima de Pramoedya, Arus Balik, que está ambientada en un periodo precolonial. Pero, desafortunadamente, no ha sido traducida. Intenta imaginar cómo era el mundo antes de la colonización holandesa, cómo era el periodo marítimo en la historia de las Indias Orientales antes de la invasión europea. Para hacerle justicia a la novela, sería necesario sumergirse en la historia del Imperio mayapajit y, antes de eso, el Imperio srivijaya, para comprender las estructuras sociopolíticas del sistema mandala, que Pramoedya recupera de una mundialización alternativa. Pero, como dije, es muy difícil escribir sobre algo que no ha sido traducido, ya que nadie ha leído el texto. Entonces hay que hacer mucha reconstrucción de la trama, etc., para los lectores, y esa no es la labor de la teoría y la crítica literaria. Max Lane, el traductor al inglés de la "tetralogía de Buru", ha intentado traducir Arus Balik durante un tiempo, pero creo que no ha acabado. Ojalá se traduzca. 
PREGUNTA: Mi pregunta conecta directamente con la anterior, porque cuando mencionó al especialista regional frente a aquel que se acerca a los textos con una perspectiva de literatura mundial, mencionó que los especialistas regionales se sumergen en el texto o imaginan sumergirse. Y me preguntaba de dónde viene la expresión «imaginan». ¿Fue algo dicho a la ligera?

PC: Lo fue, pero también obedece a mi experiencia personal. Durante muchos años la gente no supo que yo sabía chino, por lo que nunca me pidieron que escribiera sobre materiales chinos. Más tarde, en algún momento, alguien lo descubrió. Porque escribo mucho sobre cosmopolitismo, la gente empezó a pedirme que escribiera cosas nuevas para ellos. David Wang, por ejemplo, me pidió que escribiera algo para su New Literary History of Modern China. Leo esos textos porque conozco la lengua, pero no soy un especialista en historia literaria china. Incluso si eres un investigador en literatura china moderna es muy difícil si no estás bien versado en chino clásico, ya que hay tantas alusiones a textos clásicos, y la lengua literaria es muy distinta de la ordinaria. Así que me dije que me estaría engañando si pretendiera que puedo escribir sobre literatura china. Pero podría haberlo hecho, por así decirlo, porque, a menos que seas un especialista, nadie puede detectarlo la mayor parte de las veces. Pero lo que hice entonces fue decir «Sabes, estoy trabajando sobre cine chino, porque estoy escribiendo un libro sobre cine chino mundial y globalización, y te daré un capítulo que trata cine chino". Eso es lo que quise dar a entender con esa afirmación a la ligera sobre sumergirse, porque si trabajas en estudios regionales es muy fácil que te ratifiquen, en especial si eres un hablante nativo.

PREgunTA: ¿Puedo seguir con este tema? ¿Cuál es entonces el trabajo de alguien que está examinando textos con el «mundo» en mente y el de alguien que los examina con "región» en mente, y qué los diferencia?

PC: Vale, pues entonces voy a hacer que David vuelva al debate, porque creo que esto en parte proviene de la discusión que tú [David] tuviste con Gayatri [Spivak] sobre su rechazo de la literatura mundial, que Emily en parte copió. Pero ¿hay de verdad un desacuerdo? Una vez le dije a David medio en broma que probablemente él sepa más lenguas que Gayatri.

DD: Probablemente he olvidado más lenguas... Pero en todo caso ella y yo estábamos más de acuerdo de lo que podríamos haber previsto sobre este asunto. Necesitamos tanto aprender lenguas como hacer uso de la traducción, y estaría en desacuerdo con la idea de que si digo que la literatura mundial gana en traducción es el mercado estadounidense el que está hablando, ya sabéis, "dinero llama a dinero", porque dije que hay pérdidas 
y hay ganancias. Se trata en realidad de que la obra se convierte en algo distinto en traducción. Se manifiesta de una forma diferente, es fenomenológicamente distinta en otro lugar, y esto tiene que ver con su pregunta sobre el especialista regional. Porque hay una clase de comprensión del texto para el cual uno necesita conocer todos los otros textos que el autor leyó, y ese es una forma de concretar el texto. Puedo ser que uno sepa bengalí y conozca todo lo que leyó Tagore, o puede ser que uno sea medievalista que sabe italiano, latín y provenzal y sepa de todas las figuras referidas por Dante. Por otra parte, uno puede examinar a Dante en relación con Homero y Virgilio y no necesariamente necesita saberlo todo sobre sus fuentes medievales. Me enfrento muchas veces con este problema, porque a menudo trabajo sobre cosas de las que el especialista sabe de una forma diferente. En mi What Is World Literature? tengo un capítulo sobre Matilde de Magdeburgo y cómo su obra no se traduce bien entre fronteras de gen$\operatorname{der}$ (género): quienes la tradujeron temprano cambiaron totalmente lo que ella dijo, y su prosa mística pierde mucho de su valor en traducción al latín. Estaba en ese caso examinando políticas sexuadas de traducción, pero para trabajar sobre Matilde no necesito saberlo todo sobre cristología medieval; lo siento, me adormece, nunca voy a saberlo todo sobre ese tema. Así que hay distintas formas de contextualizar. Di algunas conferencias en Tokio el año pasado y hablé sobre Higuchi Ichiyō y Akutagawa Ryūnoske y Lu Xun y James Joyce como escritores que tuvieron su inicio gracias a revistas modernistas. Ninguno de ellos pretendió ser autor de relatos. Higuchi Ichiyō era poeta, y James Joyce quería escribir obras de teatro, poemas y novelas. Pero había un mercado para relatos porque había revistas, y los escritores jóvenes de la periferia del sistema pudieron acceder. Y aunque estos escritores no se conocieron entre sí, hay una semejanza en cómo funcionaban estas imprentas y qué hicieron posible. Un especialista japonés respondió a mi presentación diciendo: «Pero quiero saber acerca del contexto en el que Higuchi Ichiyō estaba escribiendo». Pero hay diversos contextos. Un contexto se compone ciertamente de los escritores japoneses que ella conocía, pero otro contexto es qué estaba pasando con esas imprentas en muchas partes del mundo y cómo contribuyeron a hacer del modernismo un fenómeno creciente a nivel mundial. Pienso que todos aquellos que trabajamos en literatura mundial debemos determinar en relación con un asunto concreto qué necesitamos saber del trabajo de los especialistas y qué no necesitamos saber, así como qué aportamos nosotros con respecto a una nueva concreción de la obra.

PC: De manera que sería justo decir que el especialista regional hace en realidad mucho menos para que el texto de un escritor sea popular. Tú, con tu enfoque sobre la literatura mundial, muy probablemente escribirías 
algo que sería más atractivo que lo de un especialista regional. Mi enfoque sobre la literatura mundial, dada mi definición de "mundo", dependería del conocimiento del especialista regional y mi trabajo sería menos atractivo para el público en general.

DD: Bien, mi discusión es que, en algunos de los campos en los que trabajo, como estudios islandeses o estudios del Oriente Próximo antiguo, los especialistas regionales dedican tanto tiempo a hacerte sentir que no puedes pensar sobre los textos si no dedicas toda tu vida a ellos, y después se preguntan por qué tienen tan pocos lectores. El otro extremo es saltar de aquí a allá y no prestar atención a su trabajo. No creo que eso sea bueno; realmente necesitas interactuar en serio con los especialistas regionales. Así que, en un mundo ideal, si estoy escribiendo sobre Matilde debo decir algo interesante también para los especialistas en Matilde, quienes pueden no haberse parado a pensar en serio por qué sus traducciones son importantes. Pero, al mismo tiempo, no voy a contribuir al estudio de la cristología medieval.

PC: Sí, pero ¿̇irías que, en cierto sentido, hay algo de resentimiento en la acusación de que tu enfoque sobre la literatura mundial es una generalización, un acercamiento más fácil? Porque a ellos les gustaría tener los tipos de lectores que tu enfoque produce.

DD: Bien, con respecto a ese término, mi hermano mayor, Leo, es un investigador muy prolífico sobre el siglo XVIII. Tenía publicados seis libros a la edad de cuarenta y cinco, y una vez le pregunté cómo lo hizo. Y me dijo: "Generalizar es la maldición de los Damrosch". Así que quizás tenga que reconocerlo, aunque la realidad es que hacen falta varios borradores para que parezca que el ensayo fue fácil de escribir. Si se escribe mal para un lector académico en general, entonces eso es generalizar; si los especialistas hacen mal su trabajo, entonces se vuelve hermético. Pienso que ambas perspectivas deben combinarse.

\section{REFERENCIAS}

ANDERSOn, Benedict.1993. Comunidades imaginadas. Reflexiones sobre el origen y la difusión del nacionalismo. Traducido por Eduardo L. Suárez. México: FCE.

ANDERSON, Benedict. 2012. The Fate of Rural Hell: Ascetism and Desire in Buddhist Thailand. Chicago: University of Chicago Press.

APTER, Emily. 2013. Against World Literature: On the Politics of Untranslatability. Londres: Verso. 
Balakian, Anna. 1959. «The Objective of Comparative Literature». En Comparative Literature: Proceedings of the Second Congress of the International Comparative Literature Association, editado por Werner P. Friederich, 1: 236-238. Chapel Hill: The University of North Carolina Press.

BALAKIAN, Anna. 1994. "How and Why I Became a Comparatist». En Building a Profession: Autobiographical Perspectives on the Beginnings of Comparative Literature in the United States, editado por Lionel Gossman y Mihai I. Spariosu, 75-87. Albany: State University of New York Press.

Casanova, Pascale. 2001. La República mundial de las Letras. Traducido por Jaime Zulaika. Barcelona: Anagrama.

CHEAH, Pheng. 2003. Spectral Nationality: Passages of Freedom from Kant to Postcolonial Literatures of Liberation. Nueva York: Columbia University Press.

CHEAH, Pheng. 2016. What Is a World? On Postcolonial Literature as World Literature. Durham, NC: Duke University Press.

CHEAH, Pheng. 2017. «Worlding Literature: Living with Tiger Spirits». Diacritics 45, n. ${ }^{\circ} 2: 86-114$.

Damrosch, David. 2003. What Is World Literature? Princeton: Princeton University Press.

DeNECKE, Wiebke. 2013. Classical World Literatures: Sino-Japanese and Greco-roman Comparisons. Oxford: Oxford University Press.

Escobar OrTiz, Jorge Manuel. 2017. «Las tablillas del país de Sumer». Huellas: Revista de la Universidad del Norte 102 (Julio-Diciembre): 57-65.

Kunniawan, Eka. 2018. Hombre Tigre. Traducido por Jacinto Pariente de Carraza. Madrid: Armaenia.

LAMPING, Dieter, ed. 2015. Meilensteine der Weltliteratur. Von der Aufklärung bis in die Gegenwart. Stuttgart: Kröner.

Lara Peinado, Federico, trad. 1988. Himnos sumerios. Madrid: Tecnos.

LÖFFLER, Sigrid. 2014. Die neue Weltliteratur und ibre großen Erzäbler. Múnich: C. H. Beck.

MARX, Karl. 2008. El proceso de producción de capital. Traducido por Pedro Scaron. Vol. 1 de El capital: crítica de la economía politica. México: Siglo XXI.

MORETTI, Franco. 2000. «Conjeturas sobre la literatura mundial». New Left Review 3 (Julio-Agosto): 65-76.

MoretTi, Franco. 2003. "Más conjeturas sobre la literatura mundial». New Left Review 20 (Mayo-Junio): 83-91.

MufTI, Aamir. 2018. Forget English! Orientalisms and World Literature. Cambridge, Mass.: Harvard University Press.

PramoJ, Kukrit. 1981. Four Reigns. Traducido por Tulachandra. Chiang Mai: Silkworm Books.

Rubio, Gonzalo. 1999. «Šulgi el políglota: del don de lenguas y la traducción en la Mesopotamia antigua». ISIMU: Revista sobre Oriente Próximo y Egipto en la Antigüedad 2: 215-222.

SANMARTín, Joaquín, trad. y ed. 2010. Epopeya de Gilgameš, rey de Uruk. Barcelona: Universitat de Barcelona / Madrid: Trotta. 
Toen, Pramoedya Ananta. 1998. La casa de cristal. Traducido por Alfonso Ormaetxea. Tafalla: Txalaparta.

Toer, Pramoedya Ananta. 2003. Tierra bumana. Traducido por Gloria Méndez. Barcelona: Destino.

Toer, Pramoedya Ananta. 2004. Hijo de todos los pueblos. Traducido por Gloria Méndez. Barcelona: Destino.

Toer, Pramoedya Ananta. 2006. Hacia el mañana. Traducido por Gloria Méndez. Barcelona: Destino.

WA Thiong'O, Ngũgĩ. 1997. Writers in Politics: A Re-Engagement with Issues of Literature and Society. Oxford: James Currey.

wa Thiong'o, Ngũgĩ. 2018. En la casa del intérprete. Traducido por Rita da Costa. Barcelona: Rayo Verde.

Ungureanu, Delia. 2017. From Paris to Tlön: Surrealism as World Literature. Nueva York: Bloomsbury.

Viswanathan, Gauri. 2015. Masks of Conquest: Literary Study and British Rule in India. Nueva York: Columbia University Press.

Warwick Research Collective [wrec]. 2015. Combined and Uneven Development: Towards a New Theory of World-Literature. Liverpool: Liverpool University Press. 
